\title{
Image Feature Extraction of Moment of Inertia Based on Otsu Threshold Segmentation
}

\author{
Qing Liu \\ School of Physics \& Information Science \\ Tianshui Normal University \\ Tianshui,Gansu,741001,China \\ xdlq@163.com
}

\author{
Liming Zhao, Lijun Zhang \\ School of Physics \& Information Science \\ Tianshui Normal University \\ Tianshui,Gansu,741001,China \\ 26489596@qq.com
}

\begin{abstract}
In order to better extract image feature and recognition, a novel feature extraction algorithm of the binary image processing using Otsu combined with normalized moment of inertia (NMI) is put forward. Firstly, the image is processed into binary image and the target area is effectively segmented utilizing Otsu algorithm based on the criteria of maximal variance between-class, secondly, the NMI feature of the binary image is extracted, and finally, the extraction NMI feature is used in image recognition. Experimental results show that NMI feature of the binary image have the ability of antigeometric distortions (translation, rotation and scaling, TRS), and anti-brightness distortions, the novel method have characteristics of simple extraction approach, little extraction parameter, easy implementation, and strong robustness.
\end{abstract}

Keywords- Image segmentation; Otsu method; Feature extraction; NMI feature

\section{INTRODUCTION}

Image recognition is an important research branch of image analysis and image understanding. It has been widely used in military, aerospace, public security and medical fields; one of the most critical technologies is the image feature extraction. In general, image feature extraction is that the physical characteristics of the target area in the image are abstracted, including the characteristics of the gray level distribution, shape and size, movement, change and sequence relations of image target. How to find the invariable features in the case of scaling, moving and rotation is one direction of the image recognition. At present, there are many kinds of image feature extraction and recognition algorithm, such as invariant moment [1], correlation method [2], projection method [3], and template identification method [4] , the normalized moment of inertia (NMI) method [5,6], etc. Those methods have already acquired certain achievements in image recognition technique, but those methods are more complex, recognition effect isn't ideal to recognize complex image, moreover, which have some limits on domain of real time image processing.

In this paper, the image is binarization processed by utilizing the Otsu Algorithm at taking fully account of the image maximum between-class variance criteria, and then the NMI invariant feature of the binary image is extracted, and further the extracted target features is used in image recognition. Finally, the validity of the proposed algorithm is verified via experiments.

\section{OTSU SEGMENTATION OF MAXIMUM BETWEEN-CLASS VARIANCE}

Otsu is a kind of image global threshold method [7]. This segmentation method is adopted to calculate the maximum variance between the target and the background rather than the histogram processed, the binary segmentation threshold is determined and the optimal segmentation threshold can be got. The segmentation principles are as follows: Gray level image is $I_{i j}$, maximum gray scale is $L$, the number of pixels is $n_{f}$ to any gray level $f$, the total pixels is $N_{0}=M \times N, t$ is the segmentation threshold of the target and background, $A_{o}$ 、 $A_{b}$ are the target and background regions. The probability and the mean can be expressed as follows

$$
\begin{gathered}
\rho_{o}=P\left(A_{o}\right)=\sum_{f=0}^{t} p_{f}=\rho(t) \\
\rho_{b}=P\left(A_{b}\right)=\sum_{f=t+1}^{L-1} p_{f}=1-\rho(t) \\
\mu_{o}=\sum_{f=0}^{t} f p_{f} / \rho_{0}=\frac{\mu(t)}{\rho(t)} \\
\mu_{b}=\sum_{f=t+1}^{L-1} f p_{f} / \rho_{b}=\frac{\mu_{\mathrm{T}}(t)-\mu(t)}{1-\rho(t)}
\end{gathered}
$$

In the above equations, $p_{f}=n_{f} / N_{0}, \mu(t)=\sum_{f=0}^{t} f p_{f}$, $\mu_{\mathrm{T}}(t)=\mu(L-1)=\sum_{f=0}^{L-1} f p_{f}$.

Then, the between-class variance of the target and the background is equation (5)

$$
\sigma^{2}=\rho_{0}\left(\mu_{0}-\mu_{\mathrm{T}}\right)^{2}+\rho_{b}\left(\mu_{b}-\mu_{\mathrm{T}}\right)^{2}=\rho_{0} \rho_{b}\left(\mu_{b}-\mu_{0}\right)
$$

The variance is the threshold function, which measures the uniform of gray distribution. The variance value is greater; the difference of the target and background in the image is also bigger. So the maximum between-class variance of segmentation means the minimize probability to be wrong segmented. Generally, $\sigma^{2}$ is selected as the 
threshold decision criterion, and then the optimal threshold $t *$ can be obtained.

$$
t^{*}=\arg \max _{0 \leq T \leq L-1} \sigma^{2}
$$

\section{IMAGE NMI FEATURE REPRESENTATION}

The following formula is defined as the center of mass of a discrete planar particle system in physics.

$$
\left.\begin{array}{l}
x_{c}=\sum x_{r} m_{r} / \sum m_{r} \\
y_{c}=\sum y_{r} m_{r} / \sum m_{r}
\end{array}\right\}
$$

$m_{r}$ is the mass of the coordinates $\left(x_{r}, y_{r}\right), \sum m_{r}$ is the total mass of the particle system. Literature $[5,6]$ introduced the concept of the center to the digital image processing. $M \times N$ pixels of two dimensional gray image can be seen as $M \times N$ particles of $\mathrm{XOY}$ plane. Pixel gray value $I_{i j}$ is consistent with the particle's mass. The image has the following definitions.

The sum of the image gray value is expressed as the image mass, it can be written with $m$.

$$
m=\sum_{i=1}^{M} \sum_{j=1}^{N} I_{i j}
$$

The concentrate points of the total mass of the image plane is defined as the center of image, it can be expressed with $\left(i_{c}, j_{c}\right)$.

$$
\left.\begin{array}{l}
i_{c}=\sum_{i=1}^{M} \sum_{j=1}^{N} i I_{i j} / \sum_{i=1}^{M} \sum_{j=1}^{N} I_{i j} \\
j_{C}=\sum_{i=1}^{M} \sum_{j=1}^{N} j I_{i j} / \sum_{i=1}^{M} \sum_{j=1}^{N} I_{i j}
\end{array}\right\}
$$

The moment of inertia of image around any given point $\left(i_{0}, j_{0}\right)$ is defined as image moment of inertia $J_{i_{0} j_{0}}$.

$$
J_{i_{0} j_{0}}=\sum_{i=1}^{M} \sum_{j=1}^{N}\left[\left(i-i_{0}\right)^{2}+\left(j-j_{0}\right)^{2}\right] I_{i j}
$$

Grayscale image, its moment of inertia is the geometric distortion and brightness distortion whether it is about its arbitrarily given point or image center point. But the moment of inertia of the binary image has good resistance characteristics to geometric distortion and brightness distortion. The normalized moment of inertia (NMI) around the center of the binary image can be defined based on the above image description.

$$
\begin{aligned}
& N M I=\sqrt{J_{i_{c} j_{c}}} / m=\sqrt{\sum_{i=1}^{M} \sum_{j=1}^{N}\left[\left(i-i_{c}\right)^{2}+\left(j-j_{c}\right)^{2}\right] I_{i j}^{\prime}} / \sum_{i=1}^{M} \sum_{j=1}^{N} I_{i j}^{\prime} \\
& =\sqrt{\sum_{i, j \in \psi} \sum\left[\left(i-i_{c}\right)^{2}+\left(j-j_{c}\right)^{2}\right]} / \sum_{i, j \in \psi} \sum I_{i j}^{\prime}
\end{aligned}
$$

$I_{i j}^{\prime}$ is the binary image, $\psi$ is the region of the value of 1 in the binary image. The NMI has feature of the small amount of calculation and extraction convenient compared with the traditional image invariant features.

\section{EXPERIMENTAL RESULTS AND ANALYSIS}

The algorithm processing step is that the grayscale image is segmented using Otsu maximum between-class variance, then the binary image divided the target is generated and the NMI invariant feature of the binary image is extracted. Finally, the algorithm is applied in image recognition.

The NMI feature extraction similarity of the binary image is represented by the distance between the images. Assuming that the identification image is expressed by A, the random image in the image database is represented by $\mathrm{B}$. NMI feature distance between the two images can be showed with the absolute value of the NMI feature difference, that is,

$$
D(A, B)=\left|N M I_{A}-N M I_{B}\right|
$$

To facilitate measurement and comparison, the equation (12) is normalized.

$$
N D(A, B)=\left|N M I_{A}-N M I_{B}\right| / N M I_{A}
$$

In order to verify the effectiveness and performance of the proposed algorithms, 100 pieces of different character images is selected as the feature recognition library and NMI feature extraction is carried out.

(1) A random image (this paper chooses Lena image) is selected to extracted the NMI feature, and the operation experiments of rotation, translation, scaling and brightness distortion is done. For Lena image, table 1 3 is the similarity error comparison of NMI extraction feature after applying this algorithm carries on the rotation, translation, and scaling and brightness distortion and with the original image features (NMI Feature extraction of image translation does not change, so it is not listed in the experimental data).

(2) NMI features are extracted to the character images in the image recognition library using this algorithm, and those feature recognition are compared with Lena Image NMI feature. Figure 1 is the portions of the character images to identify in the image library; the experimental data are shown in table 4.

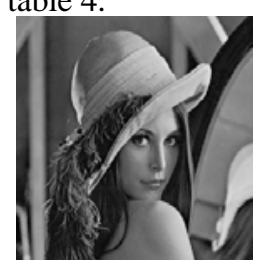

(a) Lena

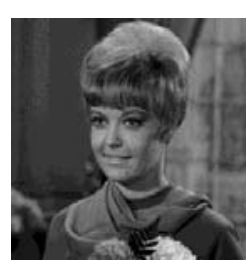

(b) Lady

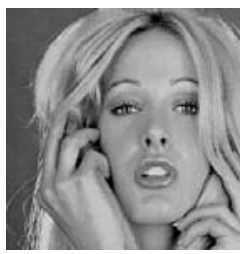

(c) Miss

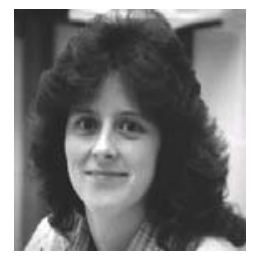

(d) Woman

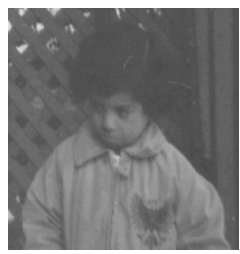

(e) Boy 


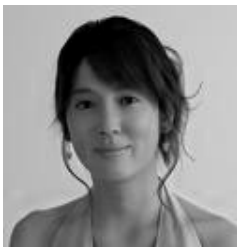

(f) Miss1

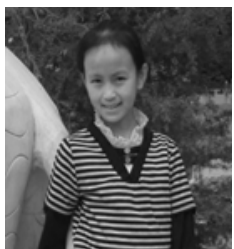

(g) Girl

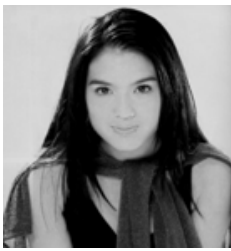

(h) Miss2
Figure 1. Portions of the character images to identify in the image library.

TABLE I. EXTRACTED NMI FEATURE AND EEOR ND WHEN SCALING FOR LENA IMAGE

\begin{tabular}{|c|c|c|}
\hline Scaling & NMI & ND (\%) \\
\hline Original Image & 0.5285 & 0.000 \\
\hline $20 \%$ & 0.5279 & 0.110 \\
\hline $40 \%$ & 0.5302 & 0.320 \\
\hline $60 \%$ & 0.5288 & 0.057 \\
\hline $80 \%$ & 0.5279 & 0.110 \\
\hline $120 \%$ & 0.5284 & 0.018 \\
\hline $140 \%$ & 0.5288 & 0.057 \\
\hline $160 \%$ & 0.5285 & 0.000 \\
\hline $180 \%$ & 0.5282 & 0.057 \\
\hline $200 \%$ & 0.5285 & 0.000 \\
\hline
\end{tabular}

TABLE II. EXTRACTED NMI FEATURE AND EEOR ND WHEN RATATION FOR LENA IMAGE

\begin{tabular}{|c|l|l|}
\hline Rotation & NMI & ND (\%) \\
\hline Original Imag & 0.5285 & 0.000 \\
\hline $10^{\circ}$ & 0.5283 & 0.037 \\
\hline $45^{\circ}$ & 0.5281 & 0.075 \\
\hline $90^{\circ}$ & 0.5285 & 0.000 \\
\hline $135^{\circ}$ & 0.5281 & 0.075 \\
\hline $180^{\circ}$ & 0.5285 & 0.000 \\
\hline $225^{\circ}$ & 0.5281 & 0.075 \\
\hline $270^{\circ}$ & 0.5285 & 0.000 \\
\hline $315^{\circ}$ & 0.5281 & 0.075 \\
\hline $330^{\circ}$ & 0.5283 & 0.037 \\
\hline & & \\
\hline
\end{tabular}

TABLE III. EXTRACTED NMI FEATURE AND EEOR ND WHEN BRIGHTNESS DISTORTION FOR LENA IMAGE

\begin{tabular}{|c|c|c|}
\hline Brightness distortion & NMI & ND (\%) \\
\hline Original Imag & 0.5285 & 0.000 \\
\hline $10 \%$ & 0.5245 & 0.760 \\
\hline $30 \%$ & 0.5259 & 0.490 \\
\hline $50 \%$ & 0.5235 & 0.950 \\
\hline $70 \%$ & 0.5285 & 0.000 \\
\hline $90 \%$ & 0.5212 & 1.380 \\
\hline $110 \%$ & 0.5212 & 1.380 \\
\hline $130 \%$ & 0.5235 & 0.950 \\
\hline $150 \%$ & 0.5259 & 0.490 \\
\hline $170 \%$ & 0.5212 & 1.380 \\
\hline
\end{tabular}

TABLE IV. EXTRACTED NMI FEATURE AND EEOR ND THAT THE DIFFERENT OF IMAGES ARE REATIVE TO LENA IMAGE

\begin{tabular}{|c|c|c|}
\hline Image & NMI & ND (\%) \\
\hline Lena & 0.5285 & 0.00 \\
\hline Lady & 0.4998 & 5.56 \\
\hline Miss & 0.4753 & 10.07 \\
\hline Woman & 0.5949 & 12.56 \\
\hline Boy & 0.4684 & 11.37 \\
\hline Miss1 & 0.6119 & 15.78 \\
\hline Girl & 0.5668 & 7.25 \\
\hline Miss2 & 0.5858 & 10.84 \\
\hline
\end{tabular}

Table 1 3 experimental results show that the proposed method not only has good geometric distortion invariant, but also has better anti brightness distortion characteristic to extract the image characteristics of NMI in the range of error $N D>\varepsilon$ ( $\varepsilon$ can be set up 2\%) when the image has certain geometric distortion and brightness distortion (TRS). The extraction of NMI can be represented and reflect the original image and the image characteristics of different distortion. In the case of default similarity identification error ( $\varepsilon=5 \%$ ) and satisfied judgment conditions, the identified images in the image library were extracted NMI characteristics and carried on image recognition and classification using the image NMI feature recognition criterion ND judgment. It obvious seen that the recognition results from the table 2 extraction image NMI feature of the images compared with Lena image in figure 1 . In addition, the other characters image recognition is also validated by experiment. These experimental results are embodied a good recognition performance and smaller error recognition rate. A lot of experiments of the feature extraction and recognition show 
that the proposed algorithm has good recognition effect for the clear image of the comparison of the target and background region. Meanwhile, it has also a certain reference to other types of image recognition.

\section{CONCLUSIONS}

According to the gray image feature extraction and target recognition, this paper proposes a feature extraction algorithm based on the combination of Otsu image binary segmentation and normalized moment of inertia. In consideration of the maximum variance between the target and background of the original image, the image is effectively segmented and target area is divided by using Otsu method, which lay the foundation for the subsequent feature extraction. And then the binary image NMI invariant features is extracted, finally, this NMI features is applied in image recognition. Experimental results show that NMI feature of the binary image have the ability of anti-geometric distortions (translation, rotation and scaling, TRS), and antibrightness distortions, the novel method have characteristics of simple extraction approach, little extraction parameter, easy implementation, and strong robustness.

\section{ACKNOWLEDGMENT}

This work is supported by Natural Science Foundation of
Gansu Province (1010RJZE028) and “QingLan” Talent Engineering Funds by Tianshui Normal University

\section{REFERENCES}

[1] Hosny, Khalid M. "On the computational aspects of affine moment invariants for gray-scale images,” Applied Mathematics and Computation, vol·195, pp.762-771,2008.

[2] Viangteeravat, Teeradache, Shirkhodaie, Amir. "Multiple target vehicles detection and classification with low-rank matrix decomposition," Proc. IEEE Symp. International Conference on System of Systems Engineering. IEEE Press, 2007, pp.1-8.

[3] Wei Zhou, Jian Guan, Guohua Zhang. "A Extracting Scheme for Targets of Interest in High Resolution Remote Sensing Imagery,” Opto-Electronic Engineering, Vol.38 pp.115-121, 2011.

[4] Pin Xu, Gui Tong, Jing Qu. "Face Detection in Video Based on AdaBoost Algorithm and Eye Location," Video Engineering, Vol.35.pp.114-118, 2011.

[5] Weng Muyun, He Mingyi. "Image Feature Detection and Matching Based on SUSAN Method,” Proceedings of the First International Conference on Innovative Computing, Information and Control. Washington, 2006, pp.322-325.

[6] Qing Liu, Luping Xu, Yide Ma,et al. "Image NMI Feature Extraction and Retrieval Method Based on Pulse Coupled Neural Networks," ACTA Automatica Sinica, Vol.36. pp. 931-938, 2010.

[7] Chao Zhang, Yan Cao, Yuncong Chen. OTSU and mutual information entropy applied in the multiple-threshold segmentation. Journal of Changchun University of Technology (Natural Science Edition), Vol. 32, pp. 57-60, 2011. 